\title{
PROJETO DE EXTENSÃO UNIVERSITÁRIA EM ENFERMAGEM MATERNO-INFANTIL: QUALITATIVOS E QUANTITATIVOS*
}

\author{
Maria Antonieta Rubio Tyrrel ** \\ Rosángela da Silva Santos ***
}

\begin{abstract}
RESUMO - Este estudo trata dos qualitativos e quantitativos do projeto de Extensão Universitária, em Enfermagem Materno-Infantil, com ênfase no Pré-Natal. São discutidos e analisados fatos e resultados da implementação das atividades, numa Unidade de Cuidados Básicos de Saúde (UCBS), por doscente e discentes de enfermagem da Escola de Enfermagem Anna Nery. A proposta de trabalho procura oferecer oportunidades para que o estudante seja capaz de administrar a assistência de enfermagem numa perspectiva de integração "estudo-trabalho" numa comunidade peri-urbana do Rio de Janeiro. Atende, também, a proposta de extensão da UFRJ Mostra a validade e importância do trabalho, a partir das avaliações dos estudantes, que participaram da implantação e de qualitativos da assistência prestada em dois anos.
\end{abstract}

ABSTRACT - This study is about the quantitative and qualitative aspects of the University Extension Program on Maternity and Child Nursing, giving emphiasis to the Pregnancy Period. Facts and results on carrying out the activities are discussed and analysed, an a Basic Care Unit (BCU), by teachers and students from the Anna Nery Nursing School. The work proposal aims of offer opportunities in order to help the student on manageing the nursing, foensing the link between "study-work", taking place in a Rio de Janeiro peripheric. It still has to do with the UFRJ extension proposal. It shows the validity and significance of this work, taking into account the students' evaluation, who took part in setting it up through a period of two years.

\section{INTRODUÇÃO}

A Escola de Enfermagem Ana Nery da UFRJ ao iniciar o empreendimento de novas "Metodologias da Enfermagem" estabeleceu abordagem inovadora na área materno-infantil com desenvolvimento de estatrégias pedagógicas numa perspectiva comunitária. Com a inclusão das diretrizes e atividades de extensão universitária, no plano atual da Reitoria da UFRJ, ensejou-se a oportunidade de empreender ampliação do ensino na área materno-infantil com efetiva participação do aluno na resolução de problemas da comunidade em que atua.

Sendo assim, o Departamento de Enfermagem Materno-Infantil, considerando as necessidades do ensino-aprendizagem e a nova política universit́ria, assumiu a organização, implementação e gerência da Unidade de Cuidados Básicos de Saúde - UCBS Materno-Infantil na Baixa do Sapateiro - Comunidade Mare.

É imperioso ressaltar que a criação dessa unidade atende à solicitação da comunidade encaminhada através da Associação de Moradores à Sub-Reitoria da Extensão e desenvolvimento - SR-5 - da UFRJ, cujo funcionamento teve início em 19.09.86, com utilização da área física colocada à disposição pela propria comunidade e apoio logístico da Divisão de Enfermagem do Hospital Universitário Clementino Fraga Filho - HUCFF/UFRJ.

\section{A SITUAÇÃO PROBLEMA}

Após aplicar por oito (08) semestres o Programa Curricular Interdepartamental - PCI XIII B, que trata do "Profissional de Enfermagem e a Obstetrícia" implementando em 1982-1986, os professores que participaram das equipes docentes eram unânimes em manifestar sua insatisfação sobre o ensino prático de enfermagem obstétrica ao cenário hospitalar. Situação que contrariava as constantes afirmativas de autoridades internacionais e nacionais e de programas governamentais que insistiam em denunciar a distorção entre a formação do enfermeiro e as necessidade da população a ser assistida. Mais grave ainda é que essa situação bloqueava o alcance das competências definidas para o graduado, na proposta curricular vigente da própria Escola Anna Nery e essas competências ficaram evidentes também na atual legislação do exercício da enfermagem obstétrica.

Dentre os aspectos que mais proucupavam os docentes e que provocavam questionamentos sistemáticos, podemos assinalar:

- as experiências curriculares, na prática se circunscreviam à parturiente na unidade hospitalar;

- o ensino limitado dos conteúdos relacionados com os problemas mais críticos da mulher por ocasião do processo de gestação, parturição e puerperio;

* Prêmio Isaura Barbosa Lima - 1 lugar - 41 Congresso Brasileiro de Enfermagem - Florianópolis - Santa Catarina.

** Professora Titular do Departamento de Enfermagem Materno-Infantil da Escola de Enfermagem Anna Nery/UFR. Autora e Coordenadora do Projeto de Extensẩo. Coordenadora Adjunta dos Cursos de Pós-Graduação em Enfermagem "Lato-Sensu" da EEAN.

*** Professora Assistente do Departamento de Enfermagem Materno-Infantil da EEAN/UFRJ. Co-autora e Sub-Coordenadora do Projeto de Extansão. Atual Chefe do Deparatamento. 
- as atividades de atendimento ao recém-nascido s6 são realizadas através dos denominados "cuidados imediatos" na sala de parto;

- as atividades de ensino guiadas por esquemas rígidos pré-estabelecidos chamados "rotinas hospitalares" não atualizadas nem discutidas;

- a orientação de todo o grupo de alunos (5-6) no centro obstétrico determinava problemas que prejudicavam não só a aprendizagem como também a qualidade da assistência à mãe e ao filho, uma vez que as múltiplas ocorrências simultâneas no centro obstétrico não permitem que um único docente possa orientar e supervisionar muitos estudantes ao mesmo tempo e garantir condições de seguranças à clientela.

Essas preocupações dos docentes eram naturalmente transmitidas aos estudantes nos debates teóricos que se chocavam com a realidade da prática. Os estudantes com sua percepção superficial pressupunham, inicialmente, que a defasagem na capacidade discente estava diretamente relacionada com a dimensão da carga horária; no entanto, a maioria dos docentes estava certa de que a solução do problema podia estar na oferta de um elenco de experiências mais adequadas ao discurso.

A reformulação de experiências curriculares conduziu os docentes à certeza de que para "habilitar" o enfermeiro, em Enfermagem Obstétrica, é indispensável assegurar modelos assistenciais que permitam ao estudante vivenciar as experiências abrangentes num modelo de trabalho que possa ser aplicado nas diversas situaçóes dos serviços de assistência materno-infantil.

Nesse sentido, foi elaborado pela autora uma proposta de trabalho que configura o projeto dse extensão universitária onde definem-se "Atividades Específicas da Enfermagem Perinatal", numa perspectiva comunitária, que completa a matriz programática que define as "Atividades Específicas na Enfermagem Perinatal", numa perspectiva hospitalar.

\section{LOCAL ONDE SE REALIZA O TRABALHO}

A comunidade da Baixa do Sapateiro é uma das dez comunidades que integram o complexo: "Favela da Maré, está localizada em Bonsucesso, no Município do Rio de Janeiro, geograficamente está entre as comunidades: Timbaú e Parque da Maré.

De acordo com o cadastramento* de 3.508 unidades, que correspomdem a $\mathbf{1 4 . 1 8 8}$ habitantes, sendo 1.508 de 19 a 24 anos, indentificaram-se 3384 chefes de famnlia. destaca-se a faixa etária por ser majoritária em todas as comunidades da região.

Dos 3.508 domićlios, 3.451 foram objeto de estudo, dos quais 3.224 são residenciais, 54 comerciais, 02 industriais, 11 de caráter comunitário e 160 de caráter misto. A descrição das unidades pelas características citadas referem como "comunitária" a Associação de Moradores, Comissão de Luz, Escolas, Templos, Posto de Saúde, Posto Policial, etc; no caráter "misto" foram consideradas residência/comércio e residência/industria.

Ao informar o grau de escolaridade, esta comuni- dade a $2^{\underline{a}}$ em ordem qualitativa, que mais registra analfabetos. Assim, foram identificados da população total de $14.188 ; 2.179$ analfabetos de 07 a 70 anos; 536 com $1^{\circ}$ grau completo, 172 com $2^{\circ}$ grau completo, 09 com grau superior completo e 38 possuem superior incompleto.

A renda familiar comprovada, tomando como base 3.384 famnlias da Baixa do Sapateiro e o salário mínimo (s.m.) de4.149,60 cruzeiros (em 17 de junho de 1980), verificou-se que nesta área constaram: 543 sem renda, 106 até 1/2s.m.; 680 de $1 / 2$ a 1 s.m.; 1.459 de 1 a 2 s.m.; 371 de 2 a 3 s.m.; 131 de 3 a 4 s.m.; 49 de 4 a 5 s.m. e 1 com maia de 10 s.m.

Foi verificado tambem que de acordo com o tempo de moradia na comunidade e situação dos imóveis: 2.704 possuem imóveis próprios, 469 são cedidos, 78 são alugados, 119 em parte são alugados, 3 têm outras situações. Do total de 3.375 chefes de famllia respondentes além de apresentar características, assinalaram que $72 \%$ (2.408) dos mesmos moram há mais de 10 anos.

Dos 16.817 Chefes de Familia da Área da Mare, somente 7.981 Chefes de Família (47.46\%) responderam a pergunta sobre o tipo de participação da população, traduzindo um grande desinteresse dos moradores e qualquer tipo de participação. $O$ total de respondentes da comunidade da Baixa do Sapateiro foi de 2.844, destes, 1.918 responderam participar em atividades comunitária (associações), 864 atividades religiosas e 62 em clubes.

\section{A UNIDADE DE CUIDADOS BÁSICOS EM SAÚDE MATERNO-INFANTIL: LOCUS ESTUDO - TRABALHO EM ENFERMAGEM}

E uma unidade simples, destinada a promover cuidados básicos de saúde materno-infantil, necessários ao bem-estar da população. Essas ações, tem a finalidade de prover ef eito na promoção da saúde, na prevenção de doenças, no tratamento precoce e oportuno das afecções mais comuns e na melhoria do meio ambiente, das mães e crianças de uma comunidade periurbana.

Adota-se nela, fundamentalmente a determinação de prioridade, proposição de alternativas de solução, o uso de tecnologia simplificada, a participação ativa da comunidade através das lideranças formais e informais da mesma, e a utilização das instituições de saúde baseada nos critérios de regionalizaçảo e níveis de complexidade na prestação de serviços.

A finalidade da UCB está diretamente relacionada à necessidade de garantir um Programa Assistencial, em uma comunidade periurbana, que permite a capitação do profissional enfermeiro, em consonância com as reais necessidades da clientela alvo.

Numa acepção ampla uma UCB, abrange aspectos que vão além dos cuidados restritos à população materno-infantil, mas refere-se a um conjunto de ações de saúde dirigido à pessoas na unidade familiar, grupos e à comunidade propriamente dita. Porém nesta primeira etapa de trabalho foram implantados cuidados considerados prioritários e estão relacionados com a saúde materno-infantil por ocasião do processo gestatorio, o preparo adequado ao parto e ao processo puerperal, incluindo também os cuidados com a criança; tudo isso, num enfoque de projeção que atinge a possibilidade de cuidados no lar.

* PROJE TO RIO-PROMORAR. Levantamento Cadastral da Área da Mare. Rio de Janeiro, 1981. 
A UCB, como experiência de extensão e de integração ESCOLA - COMUNIDADE, tem destaque em quatro relevantes aspectos: à escola permite cumprir seu papel social como unidade universitária; ao docente impõe permanente integração com os problemas de saúde que lhe asseguram a renovação da teorização na prática; ao estudante, of erece a oportunidade de vivenciar os problemas sanitários com os quais terá que atuar na vida profissional; a clientela permite participar mais ativamente das ações de saúde para equacionar seus problemas, mediante movimentos populares.

Trata-se também de uma unidade assistencial organizada, implementada e gerenciada por enfermeiras professoras. Suas atividades são totalmente desenvolvidas por discentes e docentes de enfermagem, caracterizando um modelo de enfermagem autônomo em nível de atenção primário de saúde.

Neste sentido, as aspectos refenciais teoricos, dizem respeito: a) Atenção Primária de Saúde, b) Enfoque de risco na área materno-infantil, c) Ações Básicas de Saúde orientadas para o auto-cuidado.

São objetivos específicos da UCB:

a) Corrigir as deficiências no ensino de enfermagem em nível de "Habilitação Específica em Enfermagem Obstétrica";

b) Definir estratégias de integração de estudo / trabalho, numa dinâmica de trabalho comunitário;

c) Atuar na comunidade, definindo atividades de enfermagem visando a melhoria da saúde da famnlia, atividades estas que representam seu real compromisso social com a comunidade;

d) Estabelecer mecanismos que assegurem a adoção de um sistema referência e contra-referência de saúde perinatal e de saúde materno-infantil;

e) Encaminhar oportunamente as pacientes de alto risco aos outros serviços da área da saúde que fazem parte dos programas integrados, obedecendo os critérios de regionalização e Hierarquização dos problemas;

f) Organizar um sistema de registro e anotações que sirvam de base à auditoria dos cuidados prestados e de fonte de pesquisa na área.

\section{QUALITATIVOS}

A UCB, como Projeto de Extensão Universitária em Enfermagem Materno-Infantil, tem caráter institucional e foi aprovado pelos Colegiados competentes de EEAN: Corpo Deliberativo do Departamento, Conselho do Curso de Graduação e Conselho Departamental. Também foi aprovada pela Reitoria e SubReitoria de Extensão e Desenvolvimento - SR - 2 - da UERJ, e pela Diretoria da Associação dos Moradores da Comunidade da Baixa do Sapateiro, Favela da Maré, Bonsucesso, RJ.

Constitui-se, a UCB, num cenário de prática, onde há integração do estudo, ao trabalho de Enfermagem Materno-Infantil, com as seguintes características:

a) A Escola pode imprimir no Serviço, os principios filosóficos, éticos e técnicos-científicos, que preconiza e que são necessários a formação de um enfermeiro mais "generalista" e mais "polivalente"; b) As experiências curriculares e papéis a desempenhar pelos estudantes amplia-se do cenário hospitalar para o cenário comunitário, de modo a oferecer ao formando oportunidades de exercitar-se em situações reais, que lhe permitam atingir competência necessária para a prática de assistir com a maior abrangência possível, ou seja, atendendo a gestante, parturiente, puérpera, recém-nascida e lactente na UCB e no lar;

c) As atividades de iniciação à pesquisa, dos alunos, deixam de ser limitadas, e passam a ser centradas nos problemas emergentes da prática profissional na área;

d) A interação docente-discente-cliente, alcança a sua maior abrangência no que diz respeito à melhor compreensão dos problemas de saúde no contexto social da clientela, bem como no desenvolvimento da capacidade dos estudantes no que se refere à problemática "Saúde e Sociedade".

O Enfermeiro, na sua formação, participa efetivamente integrando o estudo ao trabalho prestando serviços de enfermagem que vem ao encontro de expectativas da comunidade, e até certo ponto operacionalizam aspectos da atual política da saúde, num enfoque que busca resolver problemas reais da população materno-infantil.

O ensino de Enfermagem Materno-Infantil numa perspectiva comunitária, procura também oferecer oportunidade para que o estudante adquira competência na administração da assistência de enfermagem, numa perspectiva do trabalho comunitá J e, ainda, compatível de entender o modo de fortalecer a capacidade para decisões com a clientela, utilizando-se de um sistema regionalizado e de ações integradas de saúde tão preconizados nos discursos e tão negligenciados na prática.

Outrossim, a proposta de trabalho atende também o Programa de Extensão Universitária tão conclamada pelo Exmo Sr. Reitor da Universidade Federal do Rio de Janeiro e pela Sub-Reitoria de Extensão e Desenvolvimento - SR-2, reafirmando nesse sentido o papel inovador e criativo que cabe à Universidade no que diz respeito à formação de recursos humanos que possam responder aos anseios da sociedade e adequar-se às necessidades de saúde do país.

As ações concretas de integração do estudo ao trabalho resulta na formação de um Enfermeiro consciente do seu compromisso com a sociedade, em decorrência do custo social e econômico de sua formação profissional.

Explicitamos a seguir as atividades que se realizam na UCB e, que configuram o modelo de assistência, programas em dois (2) momentos: a curto e a longo prazo.

As atividades implantadas a curto prazo são a) consulta de enfermagem à gestante, puérpera, ao recém-nascido e ao lactente; b) encaminhamentos às instituições de apoio e referência; c) ações educativas em caráter individual e grupal; d) visita domiciliar visando: controle de absenteísmo, atendimento de pacientes de alto risco, assistência no período puérperal imediato e complementação das açōes educativas; e) cursos para gestantes, mães e escolares.

As atividades a longo prazo são: a) consulta de 
enfermagem ginecológica primária; b) consulta de enfermagem à criança pré-escolar e adolescente; c) parto domiciliar: controle de trabalho de parto, assistência à mulher no parto, assistência à mulher no pós-parto imediato, assistência ao recém-nascido; d) capacitação de parteiras empíricas: treinamento e supervisão;'e) capacitação de agentes da comunidade: treinamento e supervisão; f) cursos de educação na saúde para grupos de escolares de $1^{\circ}$ e $2^{\circ}$ graus, professores de $1^{\circ}$ e 2 - graus.

O modelo operativo de enfermagem, na assistência é, a) no pré-natal: $1^{\text {a }}$ consulta - $8^{\mathrm{a}}$ semana, $2^{\mathrm{a}}$ con-

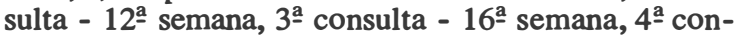
sulta - 20 semana, 5- consulta - 25- semana, 6- consulta - $30^{\mathrm{a}}$ semana, $7^{\mathrm{a}}$ consulta - $35^{\mathrm{o}}$ semana, $8^{\mathrm{a}}$ con-

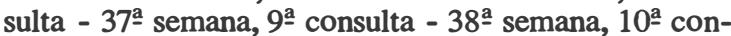
sulta - 39a semana, 11 ${ }^{\text {a }}$ consulta - se necessário; b) no pós-natal: 1- consulta - 19 semana (sete dias no parto normal e 15 dias no parto cirúrgico), $2^{\text {a }}$ consulta - 3a semana ( 21 dias), $3^{\text {a }}$ consulta - $5^{\text {a }}$ semana ( 35 dias), $4^{\text {a }}$ consulta - 2 meses ( 60 dias), 5- consulta - 3 meses (90 dias), $6^{\mathrm{a}}$ consulta -4 meses (120 dias), $7^{\mathrm{a}}$ consulta -5 meses (150 dias), 8 $8^{\text {a }}$ consulta - 6 meses (180 dias), 9 consulta - se necessário.

São procedimentos básicos nas consultas: a) da gestante e puérpera: anamnese obstétrica, exames laboratoriais, exame físico e sinais vitais, exame obstétrico (mamas, abdomem, genitália) identificação e hierarquização dos problemas, prescrições de enfermagem - ações educativas, encaminhamentos, registros das ações e evolução das situações: b) do recém-nascido e lactente: anamnese pediátrica, exame físico, monitorização do crescimento e desenvolvimento, sinais vitais, mensurações, avaliação neurológica, identificação e hierarquização dos problemas, prescrições de enfermagem - açōes educativas, encaminhamentos, registros das ações e evolução das situações; c) visita domiciliar.

A referência e a contra-referência das clientes é conforme os mecanismos formais definidos com as próprias instituições da área programática;

a) Com a X Região Administrativa da Secretaria Municipal de Saúde do Rio de Janeiro: CMS de Ramos a qual serve de apoio para as seguintes atividades: realização dos exames laboratoriais de rotina (hemograma completo, sorologia para Lues, grupo sanguíneo e $\mathrm{Rh}$, colpocitologia, EAS - urina, parasitologico, fezes, teste imunológico da gravidez - TIG. Realiza aos 60 dias após o parto um novo exame de colpocitologia e outros necessários), oferece as vacinações necessárias tanto para as gestantes quanto para as crianças, serve de referência para os cuidados médicos de atenção primária;

b) Com o Instituto de Puericultura e Pediatria Martagão Gesteira - IPPMG/UFRJ: atende a gestante, puérpera e a criança que após o diagnóstico é classificada no nível de alto risco; oferece apoio de cuidados médicos de atenção secundária e terciária;

c) Com a Divisão de Enfermagem do Hospital Universitário Clementino Fraga Filho da UFRJ: oferece apoio através dos setores de preparo, esterilização e distribuição de material permanente e de consumo, atende a gestante e/ou puérpera que apresenta intercorrências clínicas e gineco-obstétrica; oferece apoio também através de uma Auxiliar de Enfermagem e realização de exames complementares;

d) Com a Diretoria da'Associação de Moradores nos oferece: a área física, uma casa de material nobre, de dois andares, o pagamento da luz e da água, a conservação e limpeza através de um servente.

Como fonte de coleta de dados, foram utilizados primariamente alguns documentos informativos sobre o Campus Vicinal da UFRJ (Maré), da Escola de Enfermagem Anna Nery relativos ao currículo vigente, instrumentos de ensino e avaliação do PCI XIII B, instrumentos técnico-administrativos da UCB e a Tese de Concurso de Professor Titular da autora, referente a "Unidade de Cuidados Básicos de Saúde": Locus Estudo - Trabalho - Questões Curriculares de Enfermagem Materno-infantil".

Outras fontes foram utilizadas, alguns documentos do Ministério da Saúde - MS -, Ministério da educação e Cultura - MEC -, da Organização Panamericana da Saúde - OPAS/OMS, bem como as "Referências Bibliográficas" constantes deste estudo.

\section{QUANTITATIVOS}

Os dados refenciados neste trabalho dizem respeito ao período de funcionamento da UCB em fase de implantação, de 19.09.86 até 20.09.88, e embora os quantitativos com ênfase no Pré-Natal.

Os alunos que frequentam a UCB, o fazem nos semestres acadêmicos divididos em sub-grupos de 03 até no máximo de 05 alunos, com supervisão de 01 docente.

A carga horária de produção como consequência da integração do estudo ao trabalho perfaz um total de 20 horas semanais, com funcionamento às $3^{\mathbf{a}}$ e $6^{\mathrm{a}}$ feiras, de 08 às 17 horas e $4^{a}$ feira de 08 às 12:00 horas. No período de férias acadêmicas, o atendimento é realizado por aluno na condição de "Monitor de Extensão", supervisionado por um docente. Existe um período de recesso após a primeira quinzena de Dezembro, reiniciando as atividades após a primeira semana de janeiro (neste período fica na UCB uma auxiliar de enfermagem da mesma, treinada para fazer triagem e encaminhamento, funcionando também nos dias já referidos). O outro recesso, é no período do Camaval, onde é programado o mesmo esquema de atendimento referido por ocasião de Natal e Ano Novo.

Registram-se a seguir, os seguintes quantitativos importantes:

a) 47 alunos do curso de Graduação ( $8^{\circ}$ período) frequentaram a UCB, 13 (2\% semestre de 1986), 14 (1ํ semestre de 1987), 12 (10 semestre de 1988);

b) 58 alunos de pós-graduação. Em 1986 não frequentaram a UCB alunos de pós-graduação, por ser a fase de implantação de atividades em carater experimental. Em 1987 frequantaram 57 alunos, sendo 20 do Curso de especialização em Enfermagem Pediátrica, e 36 Curso de Especialização em Estimulação Essencial ao Desenvolvimento Infantil, curso em nível multiprofissional (médicos, enfermeiros, pedagogos, psicologicos, assistente social, fonoaudiologo e 
outros); e uma mestrada. Em 1988 frequentou também uma mestranda.

c) 1.562 horas de produção, contabilizando a carga horária de integração do estudo ao trabalho, a partir de 19.08.86 ate 20.08.88 (contemplando os dias e horários já referidos).

d) 2.524 atendimentos foram realizados no período de dois anos de funcionamento da UCB, destes, 251 correspondem ao ano de 1986; 1.197 ao ano de 1987 e 1.076 ao ano de 1988 . São considerados atendimentos, todas as atividades realizadas pela UCB, a saber, consultas de enfermagem (à gestante, mãe e criança), visita domiciliar, encaminhamentos (a CMS, IPPMG / UFRJ, MEC/UFRJ, HU/UFRJ) e outros (Controle de Tensão Arterial, curativos, aplicação de injeções, controle de peso, pedidos de exames, etc.)

e) Do total de atendimentos destaca-se a realização de 94 consultas em 1982, 477 em 1987 e 491 1988, perfazendo um total de 1.062 consultas de enfermagem.

f) 333 Visitas domiciliares, sendo 77 no ano de 1986, 193 no ano de 1987 e 63 de 1988.

g) 01 (um) curso para gestantes e mães, o qual foi realizado no primeiro semestre de 1987 sob a liderança de uma mestranda e assessoria da docente da UCB.

h) 01 (um) parto domiciliar, realizado em caráter de pronto atendimento, em uma multípara.

Cabe registrar que na UCB, as clientes são consideradas por grau de risco, e para efeito estatístico, como segue: Baixo risco aquelas que totalizaram 20 pontos na folha obstétrica I (Y Anexo 01); até 25 pontos, o médio risco; e, mais de 25 pontos, o alto risco. Ainda registra-se que na seleção da clientela para 0 atendimento na UCB, as clientes de médio risco ficam sob nossos cuidados apenas com agendamento de inter-concultas dependendo das necessidades. Neste sentido, verificou-se que as condições que qualificam o médio risco na UCB predominantemente, são os fatores sócio-econômicos e de saneamento. Para efeito estaístico os de médio risco somam-se aos de baixo risco, totalizando a clientela a ser atendida na UCB.

A seguir, registra-se a análise dos dados obtidos com o auxílio da Sub-Reitoria de Extensão e Desenvolvimento, SR-5/UFRJ, que computarizou os dados que constam nas tabelas em anexo, 02.

Assim, verifica-se na tabela $n-1$ que das 178 clientes que constituiram a amostra do estudo, 70,8\% encontram-se no primeiro trimestre, $12,4 \%$ no segundo trimestre e $6,7 \%$ no terceiro trimestre. Se, relacionarmos a idade gestacional com o grau de risco, constatarnos que no primeiro trimestre, $14,8 \%$ são de baixo risc1), $41,0 \%$ de médio e $14,6 \%$ do alto risco. No segundo trimestre, $2,8 \%$ de baixo risco, $7,3 \%$ de médio e $2,2 \%$ de alto risco. No terceiro trimestre, $0,6 \%$ de baixo risco, $5,1 \%$ de médio e $0,6 \%$ de alto risco. Nos dados "sem informação" chama-se atenção para as dúvidas frequentes das clientes sobre data da última menstruação, ciclos menstruais irregulares, e outros que s6 apos estudos e meios mais específicos nos levam a determinar a idade gestacional.

Desse modo, pode-se afirmar que das 178 clientes, $71,4 \%$ (baixo + médio risco) constituiram a clientela regularmente acompanhada e supervisionada no período pre-natal na UCB sendo $18,0 \%$ de alto risco e clientela atendida nas Instituições de referências da $\mathrm{X}$ RA e Hospitais da UFRJ. destaca-se ainda o fato de que se na tabela registra-se o maior perecentual para clientes no primeiro trimestre, este dado abrange os dois anos de funcionamento de unidade, porém cabe enfatizar que no segundo semestre de 1986 , e no primeiro semestre de 1987 a predominância de clientes era no $2^{\circ}$ trimestre, obtendo-se com isto um dos efeitos esperados sobre conscientização da procura do serviço de pré-natal o mais precocemente, o que sem dúvida tem sido passado, das clientes da UCB às clientes da comunidade que procuram espontâneamente a serviço.

Na tabela n- 2 observou-se que 57,3\% das clientes são multíparas (Para efeito deste estudo considera-se multípara a partir do $2^{\circ}$ parto), $31,4 \%$ são primiparas e $11,3 \%$ não consta a informação. Na relação da paridade com a idade das clientes constatou-se que, a maioria das primíparas $46,0 \%$ tem entre 18 a 25 anos, $42,5 \%$ são adolescentes (de 12 a 18 anos), 8,8\% tem entre 25 a 35 anos, não registra-se primíparas com mais de 35 anos, e $3 \%$ não consta a informação.,

Quanto às multíparas a maioria $50,0 \%$, também registra-se na clientela entre 18 a 25 anos; $41,4 \%$ tem 25 a 35 anos $4,8 \%$ são adolescentes e $3,8 \%$ com mais de 35 anos.

Chama-se a atenção para o número significativo de adolescentes na UCB, nesses dois anos de funcionamento, que levaram as autoras à adoção de atendimento específico à grupos de adolescentes num dos dias de atendimento, e a implementação e desenvolvimento do programa de estimulação essencial ao desenvolvimento humano com abrangência das atividades desde a gestação ate o 6 mês após ao parto.

Quanto às clientes adolescentes que tiveram seus filhos em condições de "prematuridade", os cuidados se fizeram extensivos ao hospital, onde se teve a oportunidade de informar aos pediatras as condições de ambiência e saneamento da familia, tendo modificado inclusive a condição de alta em 03 dos casos acompanhados. As orientações e as visitas domiciliares são mais frequentes e se fazem extensivas, com rigor, aos pais.

Outros dados importantes a registrar quanto ao atendimento das adolescentes gestantes, diz respeito ao cumprimento das mesmas no seu comparecimento às consultas; à condição de paridade que é analisada na tabela $n$ ㅇ 2 , onde verifica-se baixa incidência de cesareadas, podendo-se inferir que as orientações são eficazes quanto ao esclarecimento de sinais e sintomas que traduzem alterações, bem como ao tipo de parto desmistificando crenças e tabus sobre o mesmo.

Na tabela no 3 , observa-se que das 178 clientes, 99 foram encaminhadas na oportunidade, à matemidade; destas $74,7 \%$ de baixo risco; $13,1 \%$ de médio riscvo; $4,1 \%$ de alto risco, e $8,1 \%$ encontram-se sem informação.

Com relação ao tipo de parto, verifica-se que das 83 clientes que tiveram parto normal, 75,9\% eram de baixo risco, $10,8 \%$ de médio, $4,8 \%$ de alto risco e $8,5 \%$ sem informação. Das 14 clientes cesareadas, $64,2 \%$ eram de baixo risco, $28,6 \%$ de médio, nenhum cliente de alto risco e s6 $7,2 \%$ não possuiam informação. Quanto à utilização de instrumental, constatase que $100 \%$, ou sejam 2 clientes, classificadas como 
de baixo risco, onde houve a utilização do forceps.

Cabe registrar que se identifcou nos dados levantados e em atestados de 6́bitos, mas que não constam desta tabela 06 casos de natimorto por "axfixia perinatal" dos quais 4 foram expelidos por parto normal e 2 por cesáreas.

Estes dados confirmam a eficiência do Pre-Natal realizado com competência, pois mesmo nas clientes consideradas de alto risco, houve uma ausência na incidência de parto cirúrgico, o mesmo ocorrendo em relação aos casos de natimorto, em que de 178 casos somente 6 tiveram um final negativo por causa obstétrica biologica. É mister registrar que também verificou-se que o preparo para a amamentação durante o pré-natal, foi logrado com êxito pois a maioria das crianças, aproximadamente, $90 \%$, foram amamentadas exclusivamente com leite materno. Ofereceu-se apoio emocional e psico-biológicos às mães que tiveram seus filhos "natimorto".

Analisando-se os dados da tabela no 4, que trata sobre os encaminhamentos por instituição, segundo o grau de risco verifica-se que das $17 \%$ clientes que constituem a amostra do estudo, 112 foram encaminhadas às instituições do "Sistema Regionalizado de Saúde", como se segue: 44 ao CMS, 39 ao IPPMG (Instituto e Pediatria Martagão Gesteira), 15 a Maternidade Escola e 9 ao Hospital Universitário da UFRJ.

Destaca-se ainda que o total de encaminhamentos acima referido (112), 69,5\% foram de baixo risco; $21,4 \%$ de médio risco; $11,6 \%$ de alto risco e $4,5 \%$ sem informação.

Quanto às instituições encaminhadas, nota-se que a maioria das clientes ou seja, $75,5 \%$ são de baixo risco e foram encaminhadas ao CMS para assistência Médica Primária, em função de problemas identificados durante a Consulta de Enfermagem e na decorrência dos resultados dos exames (inflamações ginecológica, anemia, sífilis, outros); $16,3 \%$ consideradas de médio risco também foram encaminhadas ao CMS pelos mesmos motivos expostos acima; e 2,0\% do grupo de alto risco, ou seja, uma única cliente, com suspeita de tuberculose pulmonar, devido a necessidade de esclarecimento de diagnóstico e notificação compulsória.

Dos encaminhamentos valizados ao IPPMG, observa-se que $46,2 \%$ das clientes de baixo risco foram encaminhadas a tal instituição, fundamentalmente, por necessidade de receberem a solicitação de AuxílioNatalidade; $30,8 \%$ de médio risco, pelo mesmo motivo exposto acima e, para avaliação obstétrica quando necessário; e 23,1\% das clientes de alto risco, porque a instituição possui uma equipe multidisciplinar e atende as intercorrência obstétrica.

Quanto aos encaminhamentos realizados para a Maternidade Escola, verifica-se que $60 \%$ de baixo risco foram encaminhadas por ocasião do parto; $26,7 \%$ do médio risco devido e necessidade de realização de cardiotocografia - CTG e esclarecimento diagnóstico e/ou internação para acompanhamento da gestação $13,3 \%$ de alto risco necessitam de internação para realização de ultra-sonografia, cardiotocografia e avaliação obstétrica.

Cabe ressaltar que dos encaminhamentos realizados para o Hospital Universitário 66,7\% das clientes de baixo risco, o foram devido a necessidade de tratamento médico e realização de ultra-sonografia; ne- nhuma cliente de médiu risco foi encaminhada ao Hospital citado e só $11,1 \%$, ou seja uma única cliente de alto risco, necessitou de internação para controle de "tuberculose pulmonar" e "cardiopatia".

É importante esclarecer que das 5 clientes que não se tem informação sabe-se que 3 foram encaminhadas ao CMS e 2 ao Hospital Universitário, porém não há registro quanto ao motivo do encaminhamento.

Com estes dados pode-se inferir que a maioria dos encaminhamentos, refere-se a clientes de baixo risco e destina-se a CMS, caracterizando o tipo de assistência prestada pela UCB a nível primário e constituindo-se a porta de entrada ao atendimento a nível secundário e terciário.

$\mathrm{Na}$ tabela $\mathrm{n}-5$ verifica-se a relação entre os encaminhamentos realizados e o motivo dos mesmos. Observa-se que $35,7 \%$ dos encaminhamentos estão codificados como outros e foram considerados todos os encaminhamentos às instituições referenciadas dentre outros para realização de imunização e tratamento médico de sintomatologia e síndromes identificadas; $26,8 \%$ foram encaminhados e especialistas, como dermatologista, ginecologia, odontólogo, obstétra e pediatria; $22,3 \%$ em função de alto risco dos sintomas apresentados; $11,6 \%$ para cuidado médico primário e somente 3,6\% devido a sangramento e/ou hemorragia.

Deduz-se portanto, que a maioria dos encaminhamentos realizados pela UCB esta relacionado às ações básicas de saúde para proteção da mulher, pois todos os encaminhamentos codificados como outros $(35,7 \%)^{\prime}$ e especialidade $(26,8 \%)$, na realidade estão incluídos nos cuidados médicos primários e foram subdivididos para ef eitos de computação dos dados, sendo assim temos um percentual de $74,1 \%$ nesta categoria, constatando o tipo de assistência prestada pela Unidade de Cuidados Básicos. Outro dado que merece destaque, $\boldsymbol{\epsilon}$ a oportunidade que a cliente tem no pre-natal de, através de uma avaliação completa (laboratorial, pela anamnese e pelo exame físico-obstétrico), de detectar precocemente qualquer alteração e/ou intercorrência clínico-obstétrica, e ao mesmo tempo ter um tratamento oportuno.

Na tabela no 6 constata-se as monografias realizadas no período de 1986 a 1989 , pelos alunos no $8^{\circ}$ período do Curso de Graduação, como um dos requisitos à obtenção do Grau de Bacharel em Enfermagem, assim como os temas escolhidos pelos alunos.

Nestes dois anos, registra-se 15 monografias desenvolvidas na UCB; dessas, 7 foram realizadas no semestre de implantação da UCB em 1986; 5 no ano de 1987 e 3 no primeiro semestre de 1988. Dos temas escolhidos pelos alunos, verifica-se neste período que o aleitamento materno, tabagismo durante a gestação; gestação em adolescentes e planejamento familiar se apresentam como os temos mais procurados pelos alunos e são temas: assistência pré-natal, auto-cuidado durante a gestação; tipos de parto; tabus sexuais; consulta obstétrica e uso de drogas foram apenas uma única vez abordados.

Cabe ressaltar que durante o ano de 1986, em apenas um semestre se realizou um maior número de monografias justificando-se a necessidade de informações específicas para implementação das atividades programadas na UCB.

De um modo geral existe a motivação do habilitando em desenvolver a monografia na UCB, e os te- 
mas como se pode observar são emergentes da prática profissional retratanto deste modo a realidade por eles vivenciada. Enfatiza-se, também, que existe preocupação por parte dos docentes em diversificar os locais para realização das monografias porquanto o Programa Curricular de Ensino de Enfermagem Obstétrica que se desenvolve em 3 instituições diferentes: 2 hospitalares e uma comunitária.

\section{CONCLUSÕES}

A Unidade de Cuidados Básicos de Saúde, caracterizam-se pelo desenvolvimento de cuidados primários de saúde nos moldes recomendados na Conferência de Alma-Ata (1978) e pelos preceitos legais definidos na Nova Carta Magna, o que a torna identificável como Centro de Referência ao nível de atendimento primário e de formação e capacitação nesta área. Por esta razão concentra a formação do enfermeiro generalista, mediante programas de ensino e/ou assistência que visam o atendimento das necessidades de saúde da população.

A UCB garante um modelo assistencial que permite a definição de atividades e a determinação na natureza das Ações de Enfermagem Materno-Infantil. Para o ensino propicia um cenário mais adequado para a efetivação da integração do estudo ao trabalho, nupara pesquisa e estudos operacionais que asseguram o desenvolvimento das atividades de ensinar e de assistir na área de Enfermagem perinatal com a maior abrangência para os aspectos da Enfermagem Materno-Infantil.

Nas avaliações feitas pelos estudantes observamse dois pontos de vista. $\mathrm{O}$ primeiro diz respeito à avaliação da experiência e, o segundo às competência alcançadas.

Os depoimentos constantes no instrumento de avaliação dos estudantes que integram a equipe de implantação, traduzem a validade e importância da experiência adquirida de uma Unidade de Cuidados Básicos. destacam-se a relevância das oportunidades de Integração do Estudo ao Trabalho Comunitário; de assistir às mães e seus filhos na realidade do seu meio ambiente e de reconquistar um espaço de atuação profissional na comunidade. Ressalvam tamberm as oportunidades de divulgação de enfermagem como profissão.

Destaca-se a seguir o prununciamento de um dos estudantes de equipe de implantação: ma abordagem comunitária, e oferece oportunidades

"Foi muito importante para que se tivesse um contacto com a comunidade, quanto à assistência primária, Consultas de Enfermagem etc. Um campo de estágio bom, pois, coloca o aluno diante da realidade do cotidiano".

Quanto ao alcance das competências os estudantes consideram de muita utilidade para eles proprias, a oportunidade de realizar: consultas de enfermagem e gestantes puérperas e recém-nascidas, como atividades específicas do enfermeiro, visitas domiciliares e atividades educativas, assim como oportunidade de colocar em prática e aprofundar os seus conhecimentos científicos, de se relacionarem profissionalmente com as pessoas e de se auto-avaliar.

No que tange aos qualitativos, destacam-se os seguintes fatos:

a) Conscientização da clientela assistida, sobre a importância do pré-natal, como cuidado básico de proteção à saúde da mulher, com implicações na qualidade do parto e da condição de natalidade com diminuição do risco de adoecer e morrer no ciclo grávido-puerperal.

b) Os dados confirmam a eficiência do pré-natal verificando-se ausência na incidência de parto cirúrgico inclusive em clientes de alto risco.

c) A avaliação do grau de risco da cliente, conduz a precocidade na identificação, diagnóstico e intervenção oportuna, nas intercorrências clínico-obstétricas. Do mesmo modo, garante a selação da clientela potencialmente sadia e/ou de risco sócio-econômico para o seu atendimento, pelo enfermeiro, em nível primário.

d) Os encaminhamentos em caráter institucional permitem uma melhor utilização dos recursos humanos e institucionais, face ao estabelecimento de um sistema de referência e contra- referência, tomando como base a regionalização dos serviços de saúde e a hierarquização dos problemas identificados.

e) A UCB como cenário de prática: a formação e prática profissional pelo enfermeiro caracterizando assim a tão alme jada AUTONOMIA para o exercício de suas funções na ASSISTÊNCIA - ENSINO - PESQUISA. Propicia, também, a realização de estudos e pesquisas operacionais (indicação à pesquisa) pelos alunos de graduação "IN LOCUS", permitindo dessa forma identificar os reais problemas e traçar alternativas e soluçōes.

\section{REFERÊNCIAS BIBLIOGRÁFICAS}

1. BER TONI, Nora. Metodologia de aplication del enfoque de atencion materno-infantil. Publicacion Cientffica del Centro Latino-Americano de Perinatologia y Desarollo Humano. (CLAP), no 930. Montivideo, Uruguay, set. 1981.

2 BRASIL, Ministério da Saúde. Conferência Nacional de Saúde. $8^{\mathrm{a}}$ Brasflia, DF, 1987.

3 Conferência Nacional de Saúde e Direitos da Mulher. Relatório final. Brasflia, DF, 1987.

4 - Assistência integral d súde da criança: Ações Básicas. Centro de Documentaçăo do Ministério da Saúde. Braślia, DF, 1984.

5 - Assistência integral a saúde da mulher: Bases de Ações Programáticas. Centro de Documen- tação do Ministério da Saưde. Brasnlia, DF, 1984.

6 . Recursos humanos para Serviços Básicos de Saúde. Brasflia, DF, 1982.

7 Normas para identi ficacão e controle reprodutivo, obstétrico e da infertilidade no programa de assistência materno-in fantil. Centro de Documentação do Ministério da Saúde, Brasnlia, DF, 1978.

8 CALDEYRO, Barcia Roberto. Atencion peri-natal progressiva regionalizada. Documento interno. Centro Latino-Americano de Perinatologia e Desarrollo Humano. Montivideo, Uruguay, 1978.

9 CARVALHO, Vilma de et alii. Um projeto de mudança curricular no ensino de enfermagem em nfvel de graduação que favorece aos propósitos emergentes da prática profissional.Arais. XXX CBEn, Belém, PA, 1978. 
10 CONSELHO FEDERAL DE ENFERMAGEM. Direitos $d$ Saúde e Direitos d Assistência de Enfermagem. Braślia, $\mathrm{DF}, 1986$.

11 FAUNDES, Anibal et alii. Assistência Pr-eNatal. Assistência Obstétrica Prómária: quais as necessidades do Brasil. Jornal Brasileiro de Medicina (JBM) no 3 V. 52, p.38-53, marc. 1987.

12 ORGANIZAÇĀO MUNDIAL DE SAÚDE (OMS). Reunión de lideres de projetos em atencion prómária de salud materno-infantil. Montivideo, Uruguay, dezembro, 1981.

13 . Papel de la enfermeira obstétrica em la atencion materno-infantil. Informa de Enfermeria $\mathrm{n}^{\mathrm{O}}$ 19. 1977.

14 TYRREL, Maria Antonieta Rubio. Unidade de Cuidados Básicos: "LOCUS Estudo - Trabalho: Questões Curriculares em Enfermagem Materno-Infantil. Tese de concurso de Professor Titular da EEAN/UFRJ, 1988.

ANEXO № 1

\begin{tabular}{|c|c|c|c|}
\hline$\theta$ & Dados Sobre a Gestante & & Cont. \\
\hline $\begin{array}{l}\text { Idade } \\
\text { (Anos) }\end{array}$ & & $\begin{array}{c}<20 \\
20-29 \\
30-34 \\
>\quad 35 \\
\end{array}$ & \\
\hline $\begin{array}{l}\text { Paridade } \\
\text { (Para) }\end{array}$ & & $\begin{array}{|ll|}0 & 3 \\
\text { I } & 0 \\
\text { II-III } & 3 \\
\text { IV } & 4 \\
\text { V OU MAIS } & 5 \\
\end{array}$ & \\
\hline $\begin{array}{l}\text { Companheiro } \\
\text { (Especificar) }\end{array}$ & & $\begin{array}{ll}\text { SIM } & 0 \\
\text { NÃO } & 3 \\
\end{array}$ & \\
\hline $\begin{array}{l}\text { Tabagismo } \\
\text { (Início - CIG/Dia) }\end{array}$ & & $\begin{array}{l}\text { SIM } \\
\text { NÃO }\end{array}$ & \\
\hline $\begin{array}{l}\text { Espaço Interpatal } \\
\text { (Anos) }\end{array}$ & & $\begin{aligned} & 2-5 \\
< & 2 \text { OU }>5\end{aligned}$ & \\
\hline $\begin{array}{l}\text { Trabalho } \\
\text { (Especificar) }\end{array}$ & . & $\begin{array}{ll}\text { NAO OU SED } & 0 \\
\text { NÃO SED } & 3 \\
\end{array}$ & \\
\hline $\begin{array}{l}\text { Ciclos } \\
\text { (Especificar) }\end{array}$ & & $\begin{array}{|lr|}\geqslant 25 & 0 \\
>25 & 3 \\
\text { IRREGULAR } & 3 \\
\end{array}$ & \\
\hline $\begin{array}{l}\text { Altura } \\
\text { (Metro) }\end{array}$ & & $\begin{array}{ll}\geqslant 1,47 & 3 \\
<1,47 & 0\end{array}$ & \\
\hline $\begin{array}{l}\text { Nível Sócio-Econômico } \\
\text { (Renda Per Capita } \\
\text { Em Salário Mínimo) }\end{array}$ & & $\begin{array}{cc}>1 & 0 \\
1 / 3-1 & 3 \\
<1 / 3 & 4\end{array}$ & \\
\hline 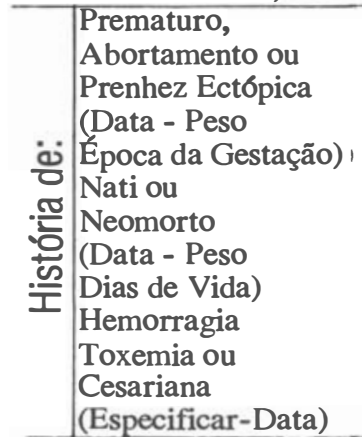 & . & $\begin{array}{lr}\text { NÃO } & 0 \\
1 & 4 \\
2 \text { OU MAIS } & 5 \\
\text { NÃO } & 0 \\
1 & 5 \\
2 \text { OU MAIS } & 11 \\
\text { NÃO } & 0 \\
\text { SIM } & 4\end{array}$ & r. \\
\hline
\end{tabular}

OBS:

20 Pontos ou mais, Risco.

Número de vezes que ficou grávida.

Número de filhos vivos no momento. 
ANEXO 2

TABELA № 1 - GEST ANTES POR GRAU DE RISCO SEGUNDO A IDADE GESTACIONAL

\begin{tabular}{|c|c|c|c|c|c|c|c|c|c|c|}
\hline \multirow{3}{*}{$\begin{array}{c}\text { Idade } \\
\text { Gestacional }\end{array}$} & \multirow{2}{*}{\multicolumn{2}{|c|}{ Total }} & \multicolumn{6}{|c|}{ Grau de Risco } & \multirow{2}{*}{\multicolumn{2}{|c|}{$\begin{array}{c}\text { Sem } \\
\text { Informação }\end{array}$}} \\
\hline & & & \multicolumn{2}{|c|}{ Baixo } & \multicolumn{2}{|c|}{ Médio } & \multicolumn{2}{|c|}{ Alto } & & \\
\hline & no & $\%$ & no & $\%$ & $\mathrm{n}^{\circ}$ & $\%$ & $n^{0}$ & $\%$ & no & $\%$ \\
\hline $1 \%$ trimestre & 126 & 70.8 & 25 & 14.0 & 73 & 41.0 & 26 & 14.6 & 2 & 1.1 \\
\hline $2 \%$ trimestre & 22 & 12.4 & 5 & 2.8 & 13 & 7.3 & 4 & 2.2 & - & 0.0 \\
\hline $3^{\circ}$ trimestre & 12 & 6.7 & 1 & 0.6 & 9 & 5.1 & 1 & 0.6 & 1 & 0.6 \\
\hline Sem informação & 18 & 10.1 & 1 & 0.6 & - & 0.0 & 1 & 0.6 & 16 & 9.0 \\
\hline Total & 178 & 100.0 & 32 & 18.0 & 95 & 53.4 & 32 & 18.0 & 19 & 10.7 \\
\hline
\end{tabular}

TABELA No 2

GESTANTES POR CONDIÇÃO DE PARIDADE SEGUNDO A IDADE CRONOLÓGICA

\begin{tabular}{|c|c|c|c|c|c|c|}
\hline \multirow[b]{2}{*}{ Idade } & \multicolumn{2}{|c|}{ Primípara } & \multicolumn{2}{|c|}{ Multípara } & \multicolumn{2}{|c|}{ Sem Informação } \\
\hline & no & $\%$ & $n^{2}$ & $\%$ & $n^{0}$ & $\%$ \\
\hline $12-15$ anos & 2 & 10.5 & 0 & 0 & 1 & 5.0 \\
\hline $15-18$ anos & 18 & 32.0 & 5 & 4.8 & 2 & 10.0 \\
\hline $18-25$ anos & 25 & 46.0 & 51 & 50.0 & 4 & 20.0 \\
\hline $25-35$ anos & 5 & 8.5 & 42 & 41.4 & 7 & 35.0 \\
\hline 35 e mais & 0 & 0 & 4 & 3.8 & 0 & 0 \\
\hline Sem informação & 2 & 3.0 & 0 & 0 & 6 & 30.0 \\
\hline Total & 56 & 100.0 & 102 & 100.0 & 20 & 100.0 \\
\hline
\end{tabular}

TABELA № 3

GESTANTE POR CONDIÇÃO DE VIDA DO RECÉM-NASCIDO E TIPO DE PARTO SEGUNDO O GRAU DE RISCO

\begin{tabular}{|c|c|c|c|c|c|c|c|c|}
\hline \multirow{5}{*}{$\begin{array}{l}\text { Grau de } \\
\text { Risco }\end{array}$} & \multirow{2}{*}{\multicolumn{8}{|c|}{$\begin{array}{l}\text { Condição de Vida } \\
\text { Nascido Vivo }\end{array}$}} \\
\hline & & & & & & & & \\
\hline & \multirow{2}{*}{\multicolumn{2}{|c|}{ Total }} & \multicolumn{6}{|c|}{ Tipo de Parto } \\
\hline & & & \multicolumn{2}{|c|}{ Normal } & \multicolumn{2}{|c|}{ Cesário } & \multicolumn{2}{|c|}{ com Instrumental } \\
\hline & $n^{\circ}$ & $\%$ & $\mathrm{n}^{\mathrm{O}}$ & $\%$ & $\mathrm{n}^{\mathrm{o}}$ & $\%$ & $\mathrm{n}^{\mathrm{o}}$ & $\%$ \\
\hline Baixo & 74 & 74.7 & 63 & 79.0 & 64.2 & 2 & 100 & \\
\hline Médio & 13 & 13.1 & 9 & 10.8 & 4 & 28.6 & 0 & 0 \\
\hline Alto & 4 & 4.1 & 4 & 4.8 & 0 & 0 & 0 & 0 \\
\hline Sem informação & 8 & 8.1 & 7 & 8.5 & 1 & 7.2 & 0 & 0 \\
\hline Total & 99 & 100.0 & 83 & 100.0 & 14 & 100.0 & 2 & 100 \\
\hline
\end{tabular}

TABELA NN 4 -ENCAMINHAMENTOS POR INSTITUIÇĀO, SEGUNDO O GRAU DO RISCO

\begin{tabular}{|c|c|c|c|c|c|c|c|c|c|c|}
\hline \multirow{3}{*}{$\begin{array}{l}\text { Grau } \\
\text { Risco }\end{array}$} & \multirow{2}{*}{\multicolumn{2}{|c|}{ Total }} & \multicolumn{8}{|c|}{$\begin{array}{l}\text { Instituição } \\
\end{array}$} \\
\hline & & & & & & MG & Mat & scola & Hos & Univ. \\
\hline & $n^{0}$ & $\%$ & $n^{0}$ & $\%$ & $n^{0}$ & $\%$ & no & $\%$ & $\mathrm{n}^{\mathbf{0}}$ & $\%$ \\
\hline Baixo & 70 & 61.5 & 37 & 75.5 & 18 & 46.2 & 9 & 60.0 & 6 & 66.7 \\
\hline Médio & 24 & 21.4 & 8 & 18.3 & 12 & 30.8 & 4 & 26.7 & 0 & 0 \\
\hline Alto & 13 & 11.6 & 1 & 2.0 & 9 & 23.1 & 2 & 13.3 & 1 & 11.1 \\
\hline Sem informação & 5 & 4.5 & 3 & 6.1 & 0 & 0.0 & 0. & 0.0 & 2 & 22.2 \\
\hline Total & 112 & 100.0 & 49 & 100.0 & 39 & 100.0 & 15 & 100.0 & 9 & 100.0 \\
\hline
\end{tabular}

TABELA No 5 - ENCAMINHAMENTOS POR MOTIVO

\begin{tabular}{l|c|c}
\hline \multirow{2}{*}{ Motivo } & \multicolumn{2}{|c}{ Encaminhamentos } \\
\cline { 2 - 3 } & $\mathrm{n}$ - & $\%$ \\
\hline Cuidado Médico Primário & 13 & 11,6 \\
Especialidades & 30 & 26,8 \\
Alto Risco & 25 & 22,3 \\
Sangramento / hemorragia & 04 & 3,6 \\
Outros & 40 & 35,7 \\
\hline Total & 112 & 100,0 \\
\hline
\end{tabular}


TABELA № 6 - MONOGRAFIAS REALIZADAS PELOS ALUNOS DO 8 PERÍODO DO CURSO DE GRADUAÇÃO, PERÍODO 1986 - 1988

\begin{tabular}{|c|c|c|c|c|c|c|c|}
\hline \multirow{3}{*}{ Temas } & & & \multicolumn{2}{|c|}{ Ano } & \multirow{2}{*}{\multicolumn{2}{|c|}{1988}} & \multirow{3}{*}{ Total } \\
\hline & \multicolumn{2}{|c|}{1986} & \multicolumn{2}{|c|}{1987} & & & \\
\hline & $\mathrm{n}^{\mathrm{o}}$ & $\%$ & $\mathrm{n}^{\mathrm{o}}$ & $\%$ & $n^{0}$ & $\%$ & \\
\hline Aleitamento materno & 1 & 14,2 & 1 & 20,0 & - & - & 2 \\
\hline Tabagismo - gestação & 2 & 28,0 & - & - & - & - & 2 \\
\hline Gestação em adolescente & 1 & 14,2 & - & - & 1 & 33,3 & 2 \\
\hline Assistência Pré-Natal & 1 & 14,2 & - & - & - & - & 1 \\
\hline Auto-cuidado-gestação & 1 & 14,2 & - & - & - & - & 1 \\
\hline Tipos de Parto & 1 & 14,2 & 1 & 20,0 & - & - & 2 \\
\hline Tabus Sexuais & - & - & 1 & 20,0 & - & - & 1 \\
\hline Plane jamento Familiar & - & - & 1 & 20,0 & 1 & 33,3 & 2 \\
\hline Consulta obstétrica & - & - & 1 & 20,0 & - & - & 1 \\
\hline Uso de drogas & - & - & - & - & 1 & 33,3 & 1 \\
\hline Total & 7 & 100 & 5 & 100 & 3 & $99,9^{*}$ & 15 \\
\hline
\end{tabular}

